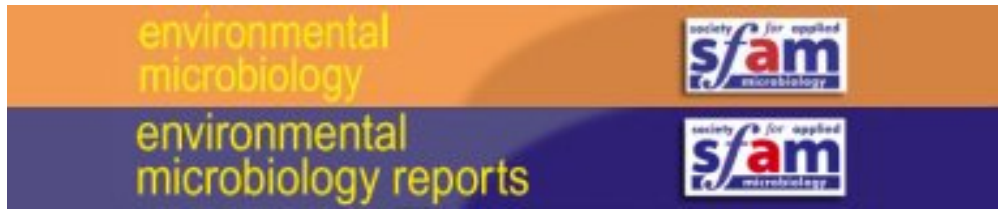

\title{
Wide diversity of parasites in Bombus terrestris (Linnaeus, 1758) revealed by a high-throughput sequencing approach
}

\begin{tabular}{|r|l|}
\hline Journal: & Environmental Microbiology and Environmental Microbiology Reports \\
\hline Manuscript ID & EMIR-2020-1828 \\
\hline Journal: & Environmental Microbiology Reports \\
\hline Manuscript Type: & EMI - Research article \\
\hline Date Submitted by the & 19-Nov-2020 \\
\hline Complete List of Authors: & $\begin{array}{l}\text { Bartolomé, Carolina; Universidade de Santiago de Compostela, CIMUS } \\
\text { Jabal-Uriel, Clara; Centro Apícola Regional, Bee Pathology } \\
\text { Buendía-Abad, María; Centro Apícola Regional, Bee Pathology } \\
\text { Benito, María; Centro Apícola Regional, Bee Pathology } \\
\text { Ornosa, Concepción; Universidad Complutense de Madrid, Departamento } \\
\text { de Biodiversidad, Ecología y Evolución } \\
\text { De la Rua, Pilar } \\
\text { Martín-Hernández, Raquel; Centro Apícola Regional, Bee Pathology } \\
\text { Higes, Mariano; Consejería de Agricultura, Junta de Comunidades de } \\
\text { Castilla-La Mancha, Bee Pathology } \\
\text { Maside, Xulio; Universidad de Santiago de Compostela, Genetics }\end{array}$ \\
\hline Keywords: & $\begin{array}{l}\text { Bombus terrestris, parasite diversity, high-throughput sequencing, } \\
\text { trypanosomatids, nosematids, neogregarines }\end{array}$ \\
\hline
\end{tabular}

\section{SCHOLARONE" Manuscripts}




\section{Wide diversity of parasites in Bombus terrestris (Linnaeus, 1758) revealed by a high-}

\section{2 throughput sequencing approach}

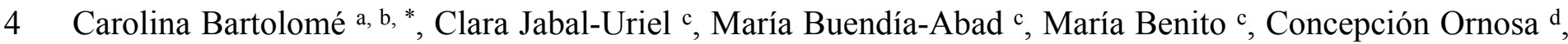
5 Pilar De la Rúa e , Raquel Martín Hernández ${ }^{\text {c, f }}$, Mariano Higes ${ }^{\text {c }}$, Xulio Maside a, b

7 a Grupo de Medicina Xenómica, CIMUS, Universidade de Santiago de Compostela, 15782 Santiago de 8 Compostela, Galicia, Spain

9 b Instituto de Investigación Sanitaria de Santiago (IDIS), 15706 Santiago de Compostela, Galicia, Spain

10 c Instituto Regional de Investigación y Desarrollo Agroalimentario y Forestal (IRIAF),

11 Laboratorio de Patología Apícola, Centro de Investigación Apícola y Agroambiental (CIAPA), Consejería 12 de Agricultura de la Junta de Comunidades de Castilla-La Mancha, 19180 Marchamalo, Spain

13 d Departamento de Biodiversidad, Ecología y Evolución, Facultad de Ciencias Biológicas, Universidad 14 Complutense de Madrid, 28040 Madrid, Spain

15 e Departamento de Zoología y Antropología Física, Facultad de Veterinaria, Universidad de Murcia, 30100 16 Murcia, Spain

$17{ }^{\mathrm{f}}$ Instituto de Recursos Humanos para la Ciencia y la Tecnología, Fundación Parque Científico Tecnológico 18 de Albacete, 02006 Albacete, Spain

20 * Corresponding author: Carolina Bartolomé; CIMUS P2D2, Av. de Barcelona s/n, Universidade de 21 Santiago de Compostela, 15782 Santiago de Compostela, Galicia, Spain; Telephone: +34 881815412; Fax: $22+34$ 8815403; email: carolina.bartolome@usc.es

24 Running tittle: Parasite diversity in bumblebees 


\section{Originality-Significance Statement}

28 High-throughput sequencing technologies enable the rapid and simultaneous identification of multiple

29 pathogens across large numbers of samples, which a priori makes them comprehensive and cost-effective 30 methods to assess parasite diversity. Here we used one of these platforms (Ion PGM ${ }^{\text {TM }}$ System) to evaluate

31 its performance in Bombus terrestris specimens that were previously PCR-identified as positive for 32 trypanosomatids (Leishmaniinae). This is the first time that the performance of classical protocols (either 33 specific PCR amplification or amplification with broad-range primers plus Sanger sequencing) and Ion 34 PGM sequencing are compared for investigating parasite diversity in bumblebees.

\section{Summary}

37 Assessing the extent of parasite diversity requires the application of appropriate molecular tools, especially 38 given the growing evidence of multiple parasite co-occurrence. Here we compared the performance of a 39 next-generation sequencing technology (Ion PGM TM System) in twelve Bombus terrestris specimens that 40 were PCR-identified as positive for trypanosomatids (Leishmaniinae) in a previous study. These bumblebees 41 were also screened for the occurrence of Nosematidae and Neogregarinorida parasites using both classical 42 protocols (either specific PCR amplification or amplification with broad-range primers plus Sanger 43 sequencing) and Ion PGM sequencing. The latter revealed higher parasite diversity within individuals, 44 especially among Leishmaniinae (which were present as a combination of Lotmaria passim, Crithidia 45 mellificae and Crithidia bombi), and the occurrence of taxa never reported in these hosts: Crithidia 46 acanthocephali and a novel neogregarinorida species. Furthermore, the complementary results produced by 47 the different sets of primers highlighted the convenience of using multiple markers to minimize the chance 48 of some target organisms going unnoticed.

49 Altogether, the deep sequencing methodology offered a more comprehensive way to investigate parasite 50 diversity than the usual identification methods and provided new insights whose importance for bumblebee 51 health should be further analysed.

\section{Introduction}


53 The drastic decline of insect populations is a matter of serious concern worldwide (Lebuhn et al., 2013; 54 Hallmann et al., 2017). Although these losses have been attributed to multiple agents, the main causes of this 55 decay are the degradation of the habitat due to urbanisation and intensive agricultural practices, the use of 56 pesticides and fertilisers, and a battery of biological factors among which are the spread of native and exotic 57 parasites (Goulson et al., 2015; Meeus et al., 2018; Sánchez-Bayo and Wyckhuys, 2019). The identification of these organisms, whose expansion in the environment is mostly driven by the sharing of foraging resources, often rely on classical techniques such as the microscopic examination of the samples (Plischuk et al., 2011; Murray et al., 2013; Graystock et al., 2015) and/ or their PCR amplification, usually followed by direct Sanger sequencing of the amplicons (Plischuk et al., 2011; Glenny et al., 2017; Jabal-Uriel et al., 2017). Although the latter is increasingly replacing microscopy due to its greater sensitivity and ability to differentiate morphologically similar organisms, it can also introduce severe biases in the analyses of prevalence and diversity of parasites. For instance, generic primers are usually designed within conserved regions of the genomes and therefore can amplify different taxa. When these occur at similar frequencies in a sample, the electropherograms exhibit overlapping peaks that may prevent the assignment of the species. On the other hand, Sanger sequencing reveals the occurrence of the predominant species, so parasites that are present at lower loads or have less amplification success (e.g. due to the occurrence of mutations in the priming sites or the sequence composition of the amplicon) can go easily undetected. These drawbacks can be partially alleviated by adding a cloning step prior to sequencing (Maharramov et al., 2013; GómezMoracho et al., 2014; Ravoet et al., 2015; Cameron et al., 2016), which is expensive and time consuming, or by performing specific multiplex PCR assays (Martín-Hernández et al., 2007; Xu et al., 2017; Bartolomé et al., 2018; Tripodi et al., 2018), which allow the detection of a limited number of targets per reaction. Although these methods have permitted the finding of several trypanosomatid (Bartolomé et al., 2018; Tripodi et al., 2018) and nosematid species (Li et al., 2012) in Bombus specimens, their technical limitations urge the use of more powerful tools to better assess the extent of parasite diversity. Nowadays, the most comprehensive and cost-effective way to investigate this matter at a large scale is to apply high-throughput sequencing methods, which have much greater depth and resolution than Sanger sequencing (Kulski, 2016) 
and enable the simultaneous identification of multiple pathogens across large numbers of samples without having to clone the amplicons.

81 The increasing evidence of trypanosomatid co-occurrence in pollinators (Ravoet et al., 2015; Bartolomé et al., 2018; Tripodi et al., 2018; Bartolomé et al., 2020) prompted us to evaluate the performance of one of these massive parallel sequencing technologies (Ion PGM TM System; Thermo Fisher Scientific Inc.) in the twelve trypanosomatid-positive bumblebees detected in Jabal-Uriel et al. (2017). These positive samples, which were identified as such by PCR amplification (Meeus et al., 2010), were also screened for the generation sequencing.

Here we report the results of this evaluation that can be considered as a test for future large-scale studies.

\section{Results and Discussion}

90 The use of Ion PGM sequencing allowed the detection of up to four species of trypanosomatids per

91 individual (Table 1), which contrasts with the finding of Crithidia bombi Lipa and Triggiani, 1988 as the only species identified by direct Sanger sequencing in the former analysis of these samples (Jabal-Uriel et al., 2017). This discordance is likely due to the fact that C. bombi is the most abundant trypanosomatid in bumblebees and masks the presence of other species poorly represented in the PCR amplification products, as shown by the "clean" electropherograms displayed by these samples (Supplemental Figure 1). Trypanosomatids appeared as a combination of three species - Lotmaria passim Evans and Schwarz, 2014, Crithidia mellificae Langridge and McGhee, 1967 and C. bombi - (Table 1). This is in good agreement with previous results from honeybee and bumblebee studies (Bartolomé et al., 2018; Bartolomé et al., 2020), and stresses the importance of applying next generation technologies to get a better overview of the parasite diversity in individual samples. This is further supported by the first detection of Crithidia acanthocephali Hanson and McGhee, 1961 in bumblebees, a protozoan that was recently found to be rather frequent in Apis mellifera Linnaeus, 1758 colonies following the same Ion PGM protocol (Bartolomé et al., 2020). A revision of taxonomic ambiguities by DNA barcoding (Boucinha et al., 2020) proved that the sequences of 
104 this trypanosomatid were identical to those of Crithidia flexonema Wallace et al., 1960, so from now on both 105 names are used for this taxon.

106 Two of the samples, B13.67 and B13.80, presented an SSU exclusive allele with a single nucleotide difference (A at position 61; Supplemental Figure 2) with respect to the sequences of Crithidia expoeki Schmid-Hempel and Tognazzo, 2010 and C. acanthocephali/ C. flexonema, and two with respect to those of C. bombi. Considering the low levels of divergence displayed by the $S S U$-locus across trypanosomatid species (Cepero et al., 2015), it was impossible to ascertain whether this sequence represented a polymorphic variant of any of the former species or corresponded to a new taxon (Trypanosomatidae sp.; Tsp in Table 1; GenBank accession numbers MW001676 and MW002685 in Supplemental Figure 2). This issue brings up the question about the importance of the selection of genes and primers in the outcome of pathogen surveys. In this case, the use of two loci (SSU and $R P B 1)$ allowed the identification of several species of trypanosomatids, although the two markers showed different sensitivities. $R P B I$ enabled the detection of L. passim, C. mellificae, C. bombi and C. acanthocephali/flexonema, whereas SSU revealed the presence of L. passim, C. bombi and the above-mentioned Trypanosomatidae sp., which illustrates the convenience of using multiple markers to minimize the chance of some target organisms going unnoticed (Supplemental Table 1). It must also be noted that this strategy also permitted to discard the presence of trypanosomatids in one of the samples initially identified as positive (B13.140) with the primers of Meeus et al. (2010).

With respect to neogregarines, the PCR-Sanger sequencing protocol detected the presence of Apicystis bombi (Liu, Macfarlane and Pengelly, 1974) in only two of the samples (B13.48 and B13.83), whereas the use of a different set of primers combined with Ion PGM sequencing permitted the detection of this species in all the specimens investigated (Table 1), as well as the identification of a new neogregarine taxon recently found in honeybees (GenBank accession number MN031271; Bartolomé et al., 2020).

The PCR detection of microsporidians revealed the occurrence of Nosema bombi Fantham and Porter, 1914 in just one individual (B13.83; Table 1); this result was confirmed by Ion PGM sequencing, which also enabled the identification of Nosema ceranae Fries, Feng, da Silva, Slemenda and Pieniazek, 1996 in this and another sample (B13.67, Table 1). It should be pointed out that although the presence of both parasites 
is regularly reported in Bombus spp. (Plischuk et al., 2009; Gamboa et al., 2015; Cameron et al., 2016;

132 Sinpoo et al., 2019), the description of different Nosema species co-occurring in single bumblebees is rather 133 rare (Li et al., 2012).

134 At any rate, it is important to emphasise that the PCR detection of a parasite in a host does not necessarily mean that it is infecting it, especially in the case of sympatric pollinators that share ecological niches and may ingest multiple pathogens deposited onto the flowers (Durrer and Schmid-Hempel, 1994; RuízGonzález et al., 2012; Graystock et al., 2015; Figueroa et al., 2019). However, regardless of their role as incidental carriers or targets of infection - whose evaluation requires the conduction of controlled experiments -, the presence of parasites in bees may contribute to spread the infection to other susceptible hosts (Evison et al., 2012; Ruíz-González et al., 2012). In this sense, improving their detection with next generation sequencing technologies might be of great help not only to refine the assessment of the levels of prevalence and parasite diversity, but also to inspire further research evaluating the potential impact that these agents may have on their hosts.

\section{Experimental Procedures}

\section{Samples and classical protocols of parasite identification}

All trypanosomatid-positive isolates (Supplemental Table 2) detected in Jabal-Uriel et al. (2017) were used to test the performance of Ion PGM sequencing for the assessment of parasite diversity. These were PCRidentified using the SEF/ SER primers of Meeus et al. (2010) and assumed to be C. bombi after Sangersequencing the amplicons of three samples (B13.39, B13.80 and B13.168).

The detection of other parasite families was also based on a preliminary PCR-screening using broad-range primers - specifically those published by Tay et al., (2005) to identify microsporidia and those designed by Meeus et al. (2010) to detect neogregarines -, followed by a further analysis to determine the species in positive samples (Jabal-Uriel et al., 2017); Nosema spp. were assigned by applying the PCR protocols described by Martín-Hernández et al. (2007) and Plischuck et al. (2009) to reveal the presence of N. ceranae and/ or $N$. apis, and that of $N$. bombi, respectively, whereas the two samples positive for neogregarines (B13.48 and B13.83) were Sanger-sequenced and identified as A. bombi. 
To perform the second part of the experiment (Ion PGM sequencing), the concentration of each DNA isolate was first measured with Nanodrop 2000 (Thermo Fisher Scientific) and set at final concentration of approximately $40 \mathrm{ng} / \mu \mathrm{l}$ for PCR-amplification with different pairs of broad-range primers.

\section{Primers and PCR amplification for further Ion PGM sequencing}

Universal primers amplifying the greatest possible number of nosematid, trypanosomatid and neogregarine species were designed with Primer Blast (https://www.ncbi.nlm.nih.gov/tools/primer-blast/) after identifying conserved regions within the alignments of Nosematidae (small-subunit ribosomal DNA - or SSU - and Actin), Leishmaniinae (SSU and RPB1-RNA polymerase II large subunit RPB1-) and Neogregarinorida (SSU) sequences available in GenBank. Amplicons were required to be shorter than 300 bp for subsequent Ion-PGM sequencing (Table 2).

PCR reactions were performed in $20 \mu \mathrm{l}$ volume containing: $10.4 \mu \mathrm{l}$ of $\mathrm{H}_{2} \mathrm{O}, 4 \mu \mathrm{l}$ of $5 \mathrm{X}$ Phusion $\mathrm{HF}$ Buffer (Thermo Fisher Scientific), $0.4 \mu \mathrm{l}$ of dNTP mix $10 \mathrm{mM}, 2 \mu \mathrm{l}$ of each primer $5 \mu \mathrm{M}, 0.2 \mu \mathrm{l}$ of Phusion HighFidelity DNA Polymerase (Thermo Fisher Scientific) and $1 \mu$ of DNA. Cycling conditions were set according to manufacturer's instructions and consisted of an initial denaturalization at $98{ }^{\circ} \mathrm{C}$ for $30 \mathrm{~s}$, followed by $35-45$ cycles of $98{ }^{\circ} \mathrm{C}$ for $10 \mathrm{~s}, 60-66{ }^{\circ} \mathrm{C}$ for $30 \mathrm{~s}$ (Table 2 ) and $72{ }^{\circ} \mathrm{C}$ for $10 \mathrm{~s}$, and a final extension of $8 \mathrm{~min}$ at $72{ }^{\circ} \mathrm{C}$. Negative controls were included in every PCR reaction, which was carried out twice to avoid biases caused by the stochastic amplification of different DNA molecules. The resulting amplicons were first checked by $2 \%$ agarose gel electrophoresis and then pooled into a single sample per Bombus specimen and marker, respectively; these were purified with Agentcourt AMPure XP (Beckman Coulter) and quantified with Qubit 2.0 (Thermo Fisher Scientific) prior to Ion PGM sequencing.

\section{Ion PGM sequencing}

\section{Library preparation and sequencing}

Amplicon Libraries were prepared at the Fundación Pública Galega de Medicina Xenómica with the Ion Plus Fragment Library Kit and the Ion Xpress Barcode Adaptors 1-96 Kit (Thermo Fisher Scientific Inc.) according to the manufacturer protocol ("Prepare Amplicon Libraries without Fragmentation Using the Ion Plus Fragment Library Kit”, Publication Number MAN0006846 Rev. B.0). Briefly, an equimolar pool of purified amplicons was prepared and a total of $100 \mathrm{ng}$ of amplified DNA per sample was used for library 
preparation. The Ion Plus Fragment Library Kit was used to end-repair the amplicons, ligate them to the Ion Xpress Barcode Adaptors and nick-repair to complete the linkage between the adapters and the inserts. Final barcoded libraries were quantified using the Ion Library TaqMan Quantitation Kit and an equimolar pool of twelve samples was prepared at a final concentration of $50 \mathrm{pM}$. Template preparation and chip loading were performed on the Ion Chef System (Thermo Fisher Scientific Inc.), whereas sequencing was carried out on the Ion PGM Sequencer using an Ion 318 ${ }^{\mathrm{TM}}$ Chip v2 (Thermo Fisher Scientific Inc.), which can deliver up to 5 million reads per run, with an average length of 200-300 base pairs (Kulski, 2016).

\section{Data analysis}

Raw reads were processed with the Ion Torrent Suite software, which sorted the data according to the barcodes assigned to each sample (Supplemental Figure 3). Afterwards, these were transformed into fasta files providing the number and sequence of the haplotypes obtained for each amplicon in a sample, as well as the number of reads on each direction. The latter was used as a control to discriminate between genuine variants and sequencing errors, as this technology tends to produce indel-strand asymmetries (Bragg et al., 2013). Thus, when a haplotype was obtained in just one direction, or when the difference between the number of forward and reverse reads was very large, it was considered artefactual. The assignment of reads to a specific amplicon was based on the sequences of the primers, which were removed from further analyses. These sequences were then aligned with Bioedit (Hall, 1999) and identified by means of BLASTn, using as search sets both the nucleotide collection (nt) and whole-genome-shotgun contigs (wgs).

All sequences obtained unintentionally (mostly from bacteria and pollen) were manually removed from further analyses.

\section{Acknowledgements}

This study was supported by the Ministerio de Economía y Competitividad (MINECO) [grant number CGL2012-34897]; Instituto Nacional de Investigación y Tecnología Agraria y Alimentaria (INIA) European Regional Development Fund (ERDF) [grant numbers RTA2014-00003-C03-01, 02 and 03]; and Fundación Séneca - Agencia de Ciencia y Tecnología de la Región de Murcia [grant of Regional Excellence 19908/GERM/2015]. We also wish to thank the authorities of the Parque Nacional de Sierra Nevada (Granada) for sampling permission. We are also grateful to B. Sobrino, N. Franjo and J. Amigo (Fundación 
211 Pública Galega de Medicina Xenómica) for their technical support as well as to A. Carracedo (Universidade

212 de Santiago de Compostela and Fundación Pública Galega de Medicina Xenómica) for the use of laboratoty 213 equipment.

\section{References}

215 Bartolomé, C., Buendía, M., Benito, M., De la Rúa, P., Ornosa, C., Martín-Hernández, R. et al. (2018) A 216 new multiplex PCR protocol to detect mixed trypanosomatid infections in species of Apis and Bombus. J $217 \quad$ Invertebr Pathol 154: 37-41.

218 Bartolomé, C., Buendía-Abad, M., Benito, M., Sobrino, B., Amigo, J., Carracedo, A. et al. (2020) 219 Longitudinal analysis on parasite diversity in honeybee colonies: new taxa, high frequency of mixed 220 infections and seasonal patterns of variation. Sci Rep 10: 10454.

221 Boucinha, C., Caetano, A.R., Santos, H.L., Helaers, R., Vikkula, M., Branquinha, M.H. et al. (2020) Analysing ambiguities in trypanosomatids taxonomy by barcoding. Mem Inst Oswaldo Cruz 115: e200504.

Bragg, L.M., Stone, G., Butler, M.K., Hugenholtz, P., and Tyson, G.W. (2013) Shining a light on dark sequencing: characterising errors in Ion Torrent PGM data. PLoS Comput Biol 9: e1003031.

Cameron, S.A., Lim, H.C., Lozier, J.D., Duennes, M.A., and Thorp, R. (2016) Test of the invasive pathogen hypothesis of bumble bee decline in North America. Proc Natl Acad Sci USA 113: 4386-4391.

Cepero, A., Martín-Hernández, R., Bartolomé, C., Gómez-Moracho, T., Barrios, L., Bernal, J. et al. (2015) Passive laboratory surveillance in Spain: pathogens as risk factors for honey bee colony collapse. J Apic Res 54: 525-531.

Durrer, S., and Schmid-Hempel, P. (1994) Shared use of flowers leads to horizontal pathogen transmission. Proc R Soc Lond B: Biol Sci 258: 299-302.

Evison, S.E.F., Roberts, K.E., Laurenson, L., Pietravalle, S., Hui, J., Biesmeijer, J.C. et al. (2012) Pervasiveness of parasites in pollinators. PLoS One 7: e30641.

Figueroa, L.L., Blinder, M., Grincavitch, C., Jelinek, A., Mann, E.K., Merva, L.A. et al. (2019) Bee pathogen transmission dynamics: deposition, persistence and acquisition on flowers. Proc Biol Sci 286: 20190603. 
Gamboa, V., Ravoet, J., Brunain, M., Smagghe, G., Meeus, I., Figueroa, J. et al. (2015) Bee pathogens found in Bombus atratus from Colombia: a case study. J Invertebr Pathol 129: 36-39.

Glenny, W., Cavigli, I., Daughenbaugh, K.F., Radford, R., Kegley, S.E., and Flenniken, M.L. (2017) Honey bee (Apis mellifera) colony health and pathogen composition in migratory beekeeping operations involved in California almond pollination. PLoS One 12: e0182814.

Gómez-Moracho, T., Maside, X., Martín-Hernández, R., Higes, M., and Bartolomé, C. (2014) High levels of genetic diversity in Nosema ceranae within Apis mellifera colonies. Parasitology 141: 475-481.

Goulson, D., Nicholls, E., Botías, C., and Rotheray, E.L. (2015) Bee declines driven by combined stress from parasites, pesticides, and lack of flowers. Science 347: 1255957.

Graystock, P., Goulson, D., and Hughes, W.O. (2015) Parasites in bloom: flowers aid dispersal and transmission of pollinator parasites within and between bee species. Proc Biol Sci 282: 20151371.

Hall, T.A. (1999) BioEdit: a user-friendly biological sequence alignment editor and analysis program for Windows 95/98/NT. Nucleic Acids Symp Ser 41: 95-98.

Hallmann, C.A., Sorg, M., Jongejans, E., Siepel, H., Hofland, N., Schwan, H. et al. (2017) More than 75 percent decline over 27 years in total flying insect biomass in protected areas. PLoS One 12: e0185809. Jabal-Uriel, C., Martín-Hernández, R., Ornosa, C., Higes, M., Berriatua, E., and De la Rúa, P. (2017) Short communication: First data on the prevalence and distribution of pathogens in bumblebees (Bombus terrestris and Bombus pascuorum) from Spain. Span J Agric Res 15: e05SC01.

Kulski, J.K. (2016) Next-generation sequencing - An overview of the history, tools, and "omic" applications. In Next generation sequencing - Advances, applications and challenges; available from: https://wwwintechopencom/books/next-generation-sequencing-advances-applications-and-challenges/nextgeneration-sequencing-an-overview-of-the-history-tools-and-omic-applications. Kulsi, J. (ed): IntechOpen. Lebuhn, G., Droege, S., Connor, E.F., Gemmill-Herren, B., Potts, S.G., Minckley, R.L. et al. (2013) Detecting insect pollinator declines on regional and global scales. Conserv Biol 27: 113-120.

Li, J., Chen, W., Wu, J., Peng, W., An, J., Schmid-Hempel, P., and Schmid-Hempel, R. (2012) Diversity of Nosema associated with bumblebees (Bombus spp.) from China. Int J Parasitol 42: 49-61. 
263 Maharramov, J., Meeus, I., Maebe, K., Arbetman, M., Morales, C., Graystock, P. et al. (2013) Genetic

264 variability of the neogregarine Apicystis bombi, an etiological agent of an emergent bumblebee disease.

$265 \quad$ PLoS One 8: e81475.

266 Martín-Hernández, R., Meana, A., Prieto, L., Salvador, A.M., Garrido-Bailón, E., and Higes, M. (2007)

267 Outcome of colonization of Apis mellifera by Nosema ceranae. Appl Environ Microbiol 73: 6331-6338.

268 Meeus, I., de Graaf, D.C., Jans, K., and Smagghe, G. (2010) Multiplex PCR detection of slowly-evolving 269 trypanosomatids and neogregarines in bumblebees using broad-range primers. J Appl Microbiol 109: 107270115.

271 Meeus, I., Pisman, M., Smagghe, G., and Piot, N. (2018) Interaction effects of different drivers of wild bee decline and their influence on host-pathogen dynamics. Curr Opin Insect Sci 26: 136-141.

Murray, T.E., Coffey, M.F., Kehoe, E., and Horgan, F.G. (2013) Pathogen prevalence in commercially reared bumble bees and evidence of spillover in conspecific populations. Biol Conserv 159: 269-276.

Plischuk, S., Meeus, I., Smagghe, G., and Lange, C.E. (2011) Apicystis bombi (Apicomplexa: Neogregarinorida) parasitizing Apis mellifera and Bombus terrestris (Hymenoptera: Apidae) in Argentina. Environ Microbiol Rep 3: 565-568.

Plischuk, S., Martín-Hernández, R., Prieto, L., Lucía, M., Botías, C., Meana, A. et al. (2009) South American native bumblebees (Hymenoptera: Apidae) infected by Nosema ceranae (Microsporidia), an emerging pathogen of honeybees (Apis mellifera). Environ Microbiol Rep 1: 131-135.

Ravoet, J., Schwarz, R.S., Descamps, T., Yañez, O., Tozkar, C.O., Martín-Hernández, R. et al. (2015) Differential diagnosis of the honey bee trypanosomatids Crithidia mellificae and Lotmaria passim. J Invertebr Pathol 130: 21-27.

Ruíz-González, M.X., Bryden, J., Moret, Y., Reber-Funk, C., Schmid-Hempel, P., and Brown, M.J. (2012) Dynamic transmission, host quality, and population structure in a multihost parasite of bumblebees. Evolution 66: 3053-3066.

Sánchez-Bayo, F., and Wyckhuys, K.A.G. (2019) Worldwide decline of the entomofauna: A review of its drivers. Biol Conserv 232: 8-27. 
289 Sinpoo, C., Disayathanoowat, T., Williams, P.H., and Chantawannakul, P. (2019) Prevalence of infection by

290 the microsporidian Nosema spp. in native bumblebees (Bombus spp.) in northern Thailand. PLoS One 14: $291 \mathrm{e} 0213171$.

Tay, W.T., O'Mahony, E.M., and Paxton, R.J. (2005) Complete rRNA gene sequences reveal that the microsporidium Nosema bombi infects diverse bumblebee (Bombus spp.) hosts and contains multiple polymorphic sites. J Eukaryot Microbiol 52: 505-513.

Tripodi, A.D., Szalanski, A.L., and Strange, J.P. (2018) Novel multiplex PCR reveals multiple trypanosomatid species infecting North American bumble bees (Hymenoptera: Apidae: Bombus). $J$ Invertebr Pathol 153: 147-155.

Xu, G., Palmer-Young, E., Skyrm, K., Daly, T., Sylvia, M., Averill, A., and Rich, S. (2017) Triplex realtime PCR for detection of Crithidia mellificae and Lotmaria passim in honey bees. Parasitol Res 117: 623628.

\section{Table legends}

Table 1. Comparison of PCR-Sanger or specific PCR vs. PCR-Ion PGM results. In the case of Ion PGM sequencing, the presence of a trypanosomatid or nosematid species in a sample was determined by a positive result $(+)$ in any of the two markers used for these parasites. Ca: Crithidia acanthocephali; $\mathrm{Cb}$ : Crithidia bombi; Cm: Crithidia mellificae; Lp: Lotmaria passim; Tsp: Trypanosomatidae sp.; Nb: Nosema bombi; Nc: Nosema ceranae; Ab: Apicystis bombi; Nsp: Neogregarinorida sp.; NA: not available.

Table 2. Primers used for PCR amplification (Ion PGM sequencing). Ta= annealing temperature, $b p=$ base 311 pairs.

\section{Supplemental Material}


315 Supplemental Table 1. Ion PGM sequencing results per locus.

316

317 Supplemental Table 2. Sampling information of the $B$. terrestris specimens analysed in this study

319 Supplemental Figure 1. Sanger-sequencing traces of Crithidia bombi obtained from bumblebee samples $320 \quad$ B13.39, B13.80 and B13.168.

322 Supplemental Figure 2. Alignment of Trypanosomatidae sp. detected in specimens B13.67 and B13.80 with 323 other trypanosomatid sequences harbouring $99 \%$ similarity at the $S S U$ gene.

325 Supplemental Figure 3. Filtering process of the Ion PGM reads. 


\begin{tabular}{|c|c|c|c|c|c|c|c|c|c|c|c|c|}
\hline \multirow[b]{3}{*}{ Specimen } & \multicolumn{6}{|c|}{ Trypanosomatids } & \multicolumn{3}{|c|}{ Neogregarines } & \multicolumn{3}{|c|}{ Nosematids } \\
\hline & \multirow{2}{*}{$\frac{\text { Sanger }}{\mathrm{Cb}}$} & \multicolumn{5}{|c|}{ Ion PGM } & \multirow{2}{*}{$\begin{array}{c}\text { Sanger } \\
\mathrm{Ab} \\
\end{array}$} & \multicolumn{2}{|c|}{ Ion PGM } & \multirow{2}{*}{$\frac{\text { Specific PCR }}{\mathrm{Nb}}$} & \multicolumn{2}{|c|}{ Ion PGM } \\
\hline & & $\mathrm{Ca}$ & $\mathrm{Cb}$ & $\mathrm{Cm}$ & $\mathrm{Lp}$ & $\overline{\mathrm{Tsp}}$ & & $\mathrm{Ab}$ & Nsp & & $\mathrm{Nb}$ & $\mathrm{Nc}$ \\
\hline B13.33 & NA & & + & + & + & & & + & + & & & \\
\hline B13.35 & NA & & + & + & + & & & + & & & & \\
\hline B13.37 & NA & + & + & + & + & & & + & & & & \\
\hline B13.39 & + & & + & + & + & & & + & & & & \\
\hline B13.44 & NA & + & + & + & + & & & + & + & & & \\
\hline B13.48 & NA & & + & + & + & & + & + & + & & & \\
\hline B13.61 & NA & & + & + & + & & & + & + & & & \\
\hline B13.67 & NA & & + & + & + & + & & + & + & & & + \\
\hline B13.80 & + & & + & + & + & + & & + & + & & & \\
\hline B13.83 & NA & & + & + & + & & + & + & & + & + & + \\
\hline B13.140 & NA & & & & & & & + & + & & & \\
\hline B13.168 & + & & + & + & + & & & + & & & & \\
\hline
\end{tabular}




\begin{tabular}{|c|c|c|c|c|}
\hline Target DNA & Primer & Sequence & Ta & Size (bp) \\
\hline Nosematid SSU & $\begin{array}{l}\text { Nos SSU-F } \\
\text { Nos SSU-R }\end{array}$ & $\begin{array}{l}\text { TGGACTGCTCAGTAATACTCACTT } \\
\text { ACTTCCCATAACTGCCTCAGA }\end{array}$ & 60 & 256 \\
\hline Nosematid Actin & $\begin{array}{l}\text { Nos Actin-F } \\
\text { Nos Actin-R }\end{array}$ & $\begin{array}{l}\text { AAGCYTGTGATGTBGATATYAGA } \\
\text { ATWGATCCACCAATCCAKACACT }\end{array}$ & 60 & 187 \\
\hline Trypanosomatid $S S U$ & $\begin{array}{l}\text { Tryp SSU-F2 } \\
\text { Tryp SSU-R2 }\end{array}$ & $\begin{array}{l}\text { GGCTACCGTTTCGGCTTTTG } \\
\text { CTTCATTCCTAGAGGCCGTG }\end{array}$ & 66 & 183 \\
\hline Trypanosomatid $R P B 1$ & $\begin{array}{l}\text { Tryp RPB1-F1 } \\
\text { Tryp RPB1-R1 }\end{array}$ & $\begin{array}{l}\text { GTGGCTGGAYCTGTGGGAGC } \\
\text { GCCRTTGATGAACTTCGCCAC }\end{array}$ & 66 & 283 \\
\hline Neogregarine $S S U$ & $\begin{array}{l}\text { Neog SSU-F } \\
\text { Neog SSU-R }\end{array}$ & $\begin{array}{l}\text { GCGCGCTACACTGATACAC } \\
\text { TTGTCCGTATTGTTCACCGGA }\end{array}$ & 64 & 222 \\
\hline
\end{tabular}

